\title{
3 Research Suare \\ Bone Defects in Tibia Managed by the Bifocal Versus Trifocal Bone Transport Technique
}

\author{
Alimujiang Abulaiti \\ the First Affiliated Hospital of Xinjiang Medical University \\ Yanshi Liu \\ the First Affiliated Hospital of Xinjiang Medical University

\section{Feiyu Cai} \\ the First Affiliated Hospital of Xinjiang Medical University \\ Kai Liu \\ the First Affiliated Hospital of Xinjiang Medical University \\ Abulaiti Abula \\ the First Affiliated Hospital of Xinjiang Medical University

\section{Xiayimaierdan Maimaiti} \\ the First Affiliated Hospital of Xinjiang Medical University

\section{Peng Ren} \\ the First Affiliated Hospital of Xinjiang Medical University \\ Aihemaitijiang Yusufu ( $\nabla$ ahmatjang@163.com ) \\ the First Affiliated Hospital of Xinjiang Medical University
}

\section{Research Article}

Keywords: bifocal bone transport, bone defect, distraction osteogenesis, trifocal bone transport, reconstruction

Posted Date: November 11th, 2021

DOl: https://doi.org/10.21203/rs.3.rs-985713/v1

License: (c) (1) This work is licensed under a Creative Commons Attribution 4.0 International License. Read Full License 


\section{Abstract}

Background: The purpose of this study was to evaluate the clinical effectiveness and determine the differences, if any, between trifocal and bifocal bone transport technique in the reconstruction of long segmental tibial bone defects caused by infection using a monolateral rail external fixator.

Methods: A total of 53 consecutive patients with long segmental tibial bone defects caused by infection and treated by monolateral rail external fixator in our department were retrospectively collected and analyzed from January 2013 to April 2019, including 39 males and 14 females with an average age of $38.8 \pm 12.4$ years (range 19 to 65 years). 32 patients were treated by bifocal bone transport (BFT) technique, and the other 21 patients were managed by trifocal bone transport (TFT) technique. The demographic data, operation duration, docking time, external fixation time, and external fixation index were documented and analyzed. Difficulties that occur during the treatment were classified according to Paley. The clinical outcomes were evaluated by the Association for the Study and Application of the Method of llizarov criteria (ASAMI) at the last clinical visit.

Results: All patients achieved an infection-free union finally, and there was no significant difference between the two groups in demographic data and both ASAMI bone and functional scores $(P>0.05)$. The mean defect size and operation duration in TFT $(9.4 \pm 1.5 \mathrm{~cm}, 161.9 \pm 8.9$ minutes) were larger than that in BFT $(7.8 \pm 1.8 \mathrm{~cm}, 122.5 \pm 11.2$ minutes $)(P<0.05)$. The mean docking time, external fixation time, and external fixation index in TFT ( $65.9 \pm 10.8$ days, $328.0 \pm 57.2$ days, $34.8 \pm 2.1$ days $/ \mathrm{cm}$ ) were all less than those in BFT (96.8 \pm 22.6 days, $474.5 \pm 103.2$ days, $60.8 \pm 1.9$ days/cm) $(\mathrm{P}<0.05)$. Difficulties and complications were more prevalent in the BFT group, while less in the TFT group $(P<0.05)$.

Conclusions: Both the trifocal and bifocal bone transport technique in the reconstruction of long segmental tibial bone defects caused by infection using a monolateral rail external fixator achieve satisfactory clinical outcomes. The trifocal bone transport technique can significantly decrease the docking time, external fixation time, external fixation index, difficulties, and complications compared with the bifocal bone transport technique.

\section{Background}

Long segmental tibial bone defects, a common clinical problem, can result from high-energy injury, the radical removal of contaminated bony fragments in an open fracture, a bone tumor resection, or repeated debridement of infected nonunion ${ }^{1,2}$. The management of this complex problem is a challenge for the treating surgeons and patients, combining with exhausting and prolonged procedures, as well as various complications $^{3-5}$. Different technical options have been proposed to reconstruct the bone defects, including acute shortening, vascularized or nonvascularized autografts, allografts, bone substitutes, and Masquelet technique ${ }^{6-13}$. However, the long duration of restricted weight-bearing, the limited source of autogenous grafts for large defects, infection, and uncertain union rates remain major concerns. 
As been widely used worldwide, the treatment of bone defects has been revolutionized so much by the bone transport technique. Lots of published data have declared this distraction osteogenesis technique is an effective and practical method for the reconstruction of large bone defects due to complete eradication of infection and powerful ability for osteogenesis ${ }^{14-20}$. The traditionally used bifocal bone transport (BFT) technique is a single-level transport with one osteotomy site, has the main drawbacks of

long frame duration, unsatisfactory regenerates, and increasing complication incidence ${ }^{15}$. The concept of trifocal bone transport (TFT), which is regarded as a double-level bone transport with two osteotomy sites, has been proposed to shorten the treatment duration for faster regeneration ${ }^{15,21-23}$. Although satisfactory clinical outcomes have been manifested by many previous researches, few data concentrated on the comparison of final clinical results and complications between the two techniques.

The purpose of this study was to evaluate the clinical effectiveness and determine the differences, if any, between trifocal and bifocal bone transport technique in the reconstruction of long segmental tibial bone defects caused by infection using a monolateral rail external fixator.

\section{Methods}

A total of 62 consecutive patients with long segmental tibial bone defects caused by infection and managed by the bone transport technique with monolateral rail external fixator (Limb Reconstruction System, LRS, Orthofix, Verona, Italy) in our department were retrospectively collected and analyzed, from January 2013 to April 2019. The inclusion criteria were: patients with tibial bone defects more than or equal to $6 \mathrm{~cm}$ treated by trifocal and bifocal bone transport technique, age older than 18 years, with a 24 months minimum follow-up. We excluded patients with pathological fracture, age older than 65 years, vascular and nerve injury, bone tumor, poor compliance, smoker, any other illness that can affect bone healing (such as diabetes), and those managed by a circular external fixator.

After exclusion criteria application, 53 patients were eligible and consisted of this study, including 53 injured limbs (left in 22, right in 31). There were 39 males and 14 females with an average age of $38.8 \pm 12.4$ years (range 19 to 65 years). Thirty-two patients were treated by the BFT, the other 21 patients were managed by the TFT. The etiology of bone defects was chronic osteomyelitis in 36 patients and infected nonunion in 17 patients. Regarding the bone defect location, there were 8 cases in the proximal $1 / 3$ of the tibial shaft, 29 cases in the middle, and 16 cases in the distal. The mean previous operation was $2.6 \pm 1.0$ times (range 1 to 5 times). Meticulous debridement of the affected tissues, installation of a monolateral rail external fixator, and a percutaneous minimally invasive cortical osteotomy using the Gigli saw were performed in all the patients. The average bone defect size after radical debridement was $8.5 \pm 1.9 \mathrm{~cm}$ (range 6 to $13 \mathrm{~cm}$ ) measured intraoperatively. There were 11 injured limbs who suffered active infection with drainage and sinus. Samples of the infected tissues were cultured, and antibiotic susceptibility tests were conducted in each patient. The culturing results manifested Staphylococcus aureus in 25 cases, Methicillin-resistant Staphylococcus aureus in 17, Pseudomonas aeruginosa in 7, and Escherichia coli in 4. 
The demographic data, operation duration (OD), docking time (DT), external fixation time (EFT), and external fixation index $(\mathrm{EFI})$ were collected and analyzed. All patients were followed up at a minimum of two years after the frame removal, and none was lost. The clinical outcomes were evaluated by the Association for the Study and Application of the Method of Ilizarov criteria (ASAMI) ${ }^{24}$ at the last followup. Difficulties that occur during the treatment were classified and documented according to Paley ${ }^{25}$. Informed consent was obtained from all patients for their data to be documented and published in our study. The Ethical Committee of our institution approved this study.

\section{Surgical procedure}

The same team performed all the surgical procedures. The patients were positioned supine on a radiolucent table under continuous general or regional anesthesia. The incision was carried down to the periosteum correspond to the previous surgical incisions when possible, protecting the healthy skin or subcutaneous tissues. The infected and devitalized bone and soft tissues were radically resected after complete hardware removal, and the samples were sent to culture for the sensitive antibiotics. The bony ends were resected until cortical bleeding, called the paprika sign ${ }^{26}$, which manifests healthy osseous tissue. Frequent alternating irrigation with hydrogen peroxide, physiological saline, and iodine liquid during and after debridement is critical.

Antibiotic-impregnated cement beads were applied for the patients with severe infection. Meticulous hemostasis was performed before wound closure. The vacuum sealing drainage (VSD) technique was used for cases with soft tissue defects or those that could not be initially closed without tension. Subsequently, local tissue flap or directly suture without tension was performed to reconstruct the small soft tissue defects, whereas flap transfer or free skin grafting was used to cover the larger wound.

Length and axis restoration of the injured extremity was achieved firstly when the monolateral external fixator was installed. Two or three Schanz screws fixed by the connecting rail were inserted on the proximal and distal bony ends, respectively. For the BFT, the other two screws were inserted on the planned transport bony fragment and fixated at the sliding block. Every screw needed to be on the same plane. According to the location of the bone defect, a percutaneous cortical osteotomy with minimal invasion was conducted using Gigli saw. The framework of TFT was similar but with two intermediate transport fragments fixated by the corresponding sliding blocks, as well as two osteotomy sites at the appropriate site. Bone defect larger than $8 \mathrm{~cm}$ or exceeded $40 \%$ of the injured bone underwent a TFT procedure ${ }^{23,33}$. When the bone defect was located at the upper or lower third tibial shaft, the two osteotomies were conducted in the longer bony segment (tandem transport). One osteotomy was performed on each side of the defect when the patient suffered a central defect (converging transport). (The detailed manipulations are shown in Figure 1).

\section{Postoperative management}

Sensitive antibiotic therapy was conducted until the infection been resolved, depending on the clinical manifestations and laboratory indicators. The small soft tissue defects were treated by direct suture or 
local tissue flap. The larger soft tissue defects were managed by free skin grafting or flap transfer.

All patients were encouraged to undergo the isometric muscle and joint range of motion (ROM) exercise on the postoperative second day, and early full-weight-bearing was also encouraged. The foot was kept in a neutral position using a rigid shoe with an elastic band, preventing ankle equines contractures. Regular pin site care was performed every day using medical alcohol or iodophors.

After a 7 days latency period, bone transport started at a rate of $0.25 \mathrm{~mm}$ in the BFT group, 4 times a day. In the TFT group, the bony fragment near the bone defect was transported $0.5 \mathrm{~mm}$ four times per day, while the other bony fragment was transported at a rate of $0.25 \mathrm{~mm}$ four times a day (tandem transport). As for converging transport, each bony fragment on both sides of the bone defect was transported $0.25 \mathrm{~mm}$ four times per day. Notably, the transport speed was modified according to the patient's tolerance and the radiological quality of the regenerate. After docking, bone transport continued for 4 or 5 days to compress the docking site, and regenerate was allowed to consolidation.

During the bone transport phase, the regular clinical visit was performed twice a month, while monthly in the consolidation period. The monolateral external fixator was dynamized and followed by removal when the satisfactory consolidation (three uninterrupted cortices appeared at the distraction zone) and docking site complete union were achieved on the standard anteroposterior (AP) and lateral radiographs. After the frame removal, a functional brace was used to prevent refracture at the docking site or bending in the distraction zone.

\section{Statistical analysis}

Statistical analysis was performed with the SPSS 22.0 (IBM Corp, USA). Independent-samples T-tests were used to analyze the continuous variables, which were expressed as the mean, standard deviation (SD), and range of the observations. The count variables were analyzed by the Chi-square or Fisher's test, expressing as a number. A statistically significant difference was set at $P<0.05$.

\section{Results}

The demographic data of the two groups are shown in Table 1, and there are no statistically significant differences $(P>0.05)$ except for the mean defect size $(P<0.05)$. The typical bifocal bone transport is shown in Figure 2-3, while trifocal bone transport is manifested in Figure 4-5.

The mean operation duration was $122.5 \pm 11.2$ minutes in the BFT group, while $161.9 \pm 8.9$ minutes in the TFT group $(P<0.05)$. The docking time in BFT was longer than that in TFT $(96.8 \pm 22.6$ days, $65.9 \pm 10.8$ days, $P<0.05)$, as well as the external fixation time (474.5 \pm 103.2 days, $328.0 \pm 57.2$ days, $P<0.05)$. All patients achieved an infection-free union finally. The average external fixation index in BFT $(60.8 \pm 1.9$ days $/ \mathrm{cm})$ was larger than that in TFT $(34.8 \pm 2.1$ days $/ \mathrm{cm})(P<0.05)$. More details are shown in Table 2 .

According to the ASAMI bone results, for the BFT, there were excellent in 13 patients, good in 15, fair in 3 , and poor in 1 . In the TFT group, there were excellent in 11 patients, good in 8, and fair in 2 . For the 
ASAMI functional results, in the BFT, there were excellent in 10 patients, good in 16, and fair in 6 . In the TFT, there were excellent in 8 patients, good in 10, and fair in 3 . There was no significant difference between the two groups in both ASAMI bone and functional scores $(P>0.05)$. (Table 3$)$

Based on Paley, there were 43 problems, 20 obstacles, and 31 complications in the BFT group. 17 problems, 8 obstacles, and 11 complications were observed in the TFT. The mean difficulties in BFT (2.9/ patient) were larger than that in TFT $(1.7 /$ patient $)(P<0.05)$. Complications were more prevalent in the BFT group (31 complications of 32 patients), while less in the TFT group (11 complications of 21 patients) $(P<0.05)$. More details are manifested in Table 4 and Table 5.

Five patients in BFT and two patients in TFT suffered deep pin tract infection and successfully managed by screw replacement and intravenous antibiotics. The axial deviation was observed in 7 cases for BFT and 3 cases for TFT. These patients underwent apparatus modification, docking site revision, and malalignment correction with regional anesthesia under image intensifier control. Three patients in BFT and one in TFT suffered soft tissue incarceration. Surgical intervention was performed to resect the interposed soft tissues, freshen the bone ends and reopen the medullary canal. Joint stiffness was observed in 16 patients ( 11 in BFT, 5 in TFT) and successfully treated by a surgical release. Four patients in BFT suffered delayed union, and the "accordion maneuver" technique contributed to satisfactory outcomes. One patient developed nonunion in the BFT group and finally achieved bone union by autogenous iliac crest bone grafting. None of the neurovascular injury, psychological problems, and refracture were observed in the 53 patients.

\section{Discussion}

The ultimate destination of treating bone defects is to reconstruct a fully functional extremity without any unacceptable deformities or limb length discrepancy ${ }^{24}$. There is no doubt that the treatment of a long segmental bone defect is a challenge for orthopedic surgeons, especially combined with deep infection. For most treating surgeons, simultaneously managing bone defects as necessary as infection. Options for the treatment of bone defects are varied ${ }^{6-13}$, while the subsequent outcomes are not completely satisfactory. The concept of distraction osteogenesis (DO) given by llizarov, in which bone regeneration occurs when subjected to tensile stress, contributes to manage this complex problem ${ }^{27}$.

At present, the two common techniques for long bone defects are acute shortening followed by relengthening (AST) and bone transport using an external fixator. However, neurovascular injury, limb discrepancy, and blood circulation obstacles are worth considering problems when the AST is conducted on cases with bone defects $>5 \mathrm{~cm}^{9}, 14,28$. The bone transport technique has been widely applied to manage bone defects for decades ${ }^{14,15,17-20}$, and problems including infection, deformity, joint stiffness, or limb discrepancy can be resolved simultaneously. The main drawback of the bone transport technique is the long procedure in a cumbersome frame, resulting in inconvenient daily life and increasing numerous complications, including pin tract problems, joint stiffness, pain, and psychological symptoms. These problems have been the obstacles to the extended application of bone transport. In addition, many 
patients with bone defects caused by an infection usually underwent several previous operations that fail and developed compromised surrounding soft tissues. Therefore, a shorter duration and fewer complications treatment is really a goal worth striving for.

Several methods have been developed to decrease the external fixation index, reduce the potential complications, and accomplish satisfactory clinical results. S.Gupta et al. ${ }^{29}$ performed a three-stage treatment in 14 consecutive patients with tibial infected nonunion using a long lateral locked plate and a six-pin monorail fixator. In this group of patients, the mean defect size was $6.4 \mathrm{~cm}$ and the mean external fixator index was 21.2 days $/ \mathrm{cm}$, while the complication rate was 0.5 per patient. They declared that composite fixation reduces the fixator time and the associated complications for patients with a segmental tibial defect due to infected nonunion, and provides high emotional acceptance of final clinical outcomes. Gulabi.D et al. ${ }^{30}$ developed a technique to reduce complications by using a circular external fixator combined with an intramedullary nail to achieve union, limb lengthening, and regenerate stability. In their treatment of five tibial nonunion patients with bone defect, all cases acquired satisfactory outcomes. Similarly, Kocaoglu.M et al. ${ }^{31}$ also summarized their experience with distraction osteogenesis in the management of bone defects and limb-shortening due to radical debridement of chronic osteomyelitis using an external fixator combined with an intramedullary nail. Although the mentioned techniques above enable less duration in the external fixator, the combination can not accelerate the regenerate consolidation and has difficulty in resolving the chronic infection simultaneously. Furthermore, the external combined with internal fixation treatment requires several additional surgical interventions. It is difficult to accept for most patients due to their unpleasant experience in numerous previous operations.

The trifocal bone transport technique was proposed to accelerate the defect closure and shorten the treatment duration. Considering the biological characteristic of incapable speed up lengthening at just one osteotomy site, the TFT was derived from the theoretical basis of distraction simultaneously using two osteotomy leads to effectively double lengthening speed. In this procedure, the regeneration time is theoretically decreased to $50 \%$, and the total treating time is reduced accordingly ${ }^{24}$. Additionally, the regenerate consolidation time is inversely proportional to the bone defect length, two shorter fragments are therefore capable of rapid consolidation compared with only one fragment.

Borzunov ${ }^{23}$ conducted an experimental comparison in the treatment of tibial defects using BFT and multilevel technique firstly, subsequently extend to clinical application. For the management of patients with large tibial defects that ranged from 12 to $14 \mathrm{~cm}$ using the multilevel techniques, distraction duration can be reduced 2.5 times, and fixation period can be reduced between 1.3 to 1.9 times. Sala et al. ${ }^{32}$ compared the clinical outcomes of TFT and BFT in the treatment of postinfectious segmental tibial bone defects with a combined Ilizarov and Taylor spatial frame method. In their retrospective study of 12 patients with atrophic tibial nonunions, although the average lengthening size was increased $(9.7 \mathrm{~cm}$ in TFT, $5.5 \mathrm{~cm}$ in BFT) in the TFT group, there was a shorter mean period in the frame ( 379 days in TFT, 457 
in $\mathrm{BFT}$ ). In addition, the TFT technique reduced the mean lengthening index (1.31 months/cm in TFT, 2.63 months/cm in BFT).

In the present study, the clinical results of 53 patients with long segmental tibial bone defects treated by bifocal or trifocal bone transport technique were retrospectively analyzed and compared. The mean defect size was significantly increased in the TFT group $(9.4 \pm 1.5 \mathrm{~cm}$ in TFT, $7.8 \pm 1.8 \mathrm{~cm}$ in BFT). In contrast, the mean docking time ( $65.9 \pm 10.8$ days in TFT, $96.8 \pm 22.6$ days in BFT), mean external fixation time (328.0 \pm 57.2 days in TFT, $474.5 \pm 103.2$ days in BFT), and mean external fixation index $(34.8 \pm 2.1$ days/cm in TFT, $60.8 \pm 1.9$ days/cm in BFT) were significantly reduced. The results were comparable to the previous studies ${ }^{23,32}$.

A previous study reported that hypoplastic bone formation was a common complication when the bone defects exceed $5 \mathrm{~cm}$ or $40 \%$ of the injured bone when treating by $\mathrm{BFT}^{33}$. The regenerate consolidation will be affected by the osteotomy technique and location, distraction length, and blood supply of transported fragment. In our study, there were 4 patients who suffer delayed consolidation in BFT, and none was observed in the TFT group. According to our experience, low energy osteotomy technique, timely lengthening speed adjustment, and application of TFT technique in massive bone defects were recommended for avoiding this problem.

Although additional surgical procedures of one transport sliding block and an osteotomy site in TFT may increase the complications correspond to Schanz screws and distraction zone, the faster regeneration and early frame removal have counteracted this negative effect. In this study, the BFT group had a statistically significant increase in mean rate of difficulty (2.9 difficulties/patient in BFT, 1.7 difficulties/patient in TFT) and complication ( 1.0 complications per patient in BFT, 0.5 complications per patient in TFT), this can be explained by the longer frame duration in these patients. The only shortcoming of the TFT group is the significantly increasing operation time ( $161.9 \pm 8.9$ minutes in TFT, $122.5 \pm 11.2$ minutes in $\mathrm{BFT})$.

Furthermore, compare with previous studies ${ }^{15,28,32}$, the llizarov circular external fixator is relatively cumbersome, complex, difficult-learning, time-consuming, and fraught with lots of potential complications. The monolateral rail external fixator used in this study, working on the same principles as Ilizarov circular external fixator, is portable, easy to construct, and has a short learning curve. It is also easy to adjust the sliding clamps adapt to the transporting bone fragments without altering the nut bolts over the threaded rods, unlike in the llizarov circular external fixator.

The clinical results of the present study manifest that both BFT and TFT in the reconstruction of long segmental tibial bone defects caused by infection using a monolateral rail external fixator achieve satisfactory outcomes. Although there were no significant differences between BFT and TFT in the final bone and functional results, the external fixation index, difficulties, and complications were significantly decreased in the TFT group. According to our experience, the most vital step is radical debridement of the infectious tissues in the reconstruction of bone defects caused by infection. In addition, comprehensive 
frame understanding, prudent patient selection, appropriate pins insertion, meticulous care, early complications detection, and proper intervention or psychological counseling ensure satisfactory results.

The present study may be limited by the retrospective nature with a single-center small sample size, a conservative attitude therefore should be adopted regarding the interpretations of our results. Multicentered trials with larger sample size, life quality assessments, and mental evaluations should be adopted in further investigations.

\section{Conclusion}

Both the trifocal and bifocal bone transport technique in the reconstruction of long segmental tibial bone defects caused by infection using a monolateral rail external fixator achieve satisfactory clinical outcomes. The trifocal bone transport technique can significantly decrease the docking time, external fixation time, external fixation index, difficulties, and complications compared with the bifocal bone transport technique.

\section{Abbreviations}

BFT: bifocal bone transport

TFT: trifocal bone transport

OD: operation duration

DT: docking time

EFT: external fixation time

EFI: external fixation index

ASAMI: the Association for the Study and Application of the Method of llizarov criteria

VSD: vacuum sealing drainage

ROM: range of motion

AP: anteroposterior

DO: distraction osteogenesis

\section{Declarations}

\section{Ethics approval and consent to participate}


All methods in this study were carried out in accordance with the Declaration of Helsinki. This study was approved by the Ethics Committee of The First Affiliated Hospital of Xinjiang Medical University. Written informed consent was obtained from all patients for their data to be recorded in our study.

\section{Consent for publication}

Informed consent was obtained from all patients for their data to be published in our study.

\section{Availability of data and materials}

The datasets analysed during the current study are available from the corresponding author on reasonable request.

\section{Competing interests}

The authors declare that they have no competing interests.

\section{Funding}

This study was funded by the grants from National Natural Science Foundation of China (No. 82060401), and the Supportive Fundation of Clinical Peak Discipline of Xinjiang Medical University (No.330104006020801). The funding body was involved in the collection, analysis, and interpretation of data by supporting with salary for the time needed. They were not involved in the design or writing the manuscript.

\section{Authors' Contributions}

AA: Conducted the study. Collected, analyzed and interpreted the data. Wrote the manuscript. YSL: Collected, interpreted and analyzed the data. Edited the manuscript. FYC: Created and statistical analyzed the data. Edited the manuscript. KL: Provided software assistance. Edited the manuscript. AA: Conducted the study and provided the data. XM: Conducted the study and provided the data. PR: Conducted the study and provided the data. AY: Planned the project. Reviewed the manuscript. All authors read and approved the final manuscript.

\section{Acknowledgements}

Not applicable

\section{References}

1. Reichert JC, Saifzadeh S, Wullschleger ME, Epari DR, Schutz MA, Duda GN, et al. The challenge of establishing preclinical models for segmental bone defect research. BIOMATERIALS 2009;30:2149-63.

2. Sadek AF, Laklok MA, Fouly EH, Elshafie M. Two stage reconstruction versus bone transport in management of resistant infected tibial diaphyseal nonunion with a gap. Arch Orthop Trauma Surg 
3. Azzam W, Atef A. Our experience in the management of segmental bone defects caused by gunshots. INT ORTHOP 2016;40:233-8.

4. Eralp L, Kocaoglu M, Rashid H. Reconstruction of segmental bone defects due to chronic osteomyelitis with use of an external fixator and an intramedullary nail. Surgical technique. J BONE JOINT SURG AM 2007;89 Suppl 2 Pt.2:183-95.

5. DeCoster TA, Gehlert RJ, Mikola EA, Pirela-Cruz MA. Management of posttraumatic segmental bone defects. J Am Acad Orthop Surg 2004;12:28-38.

6. Masquelet AC, Begue T. The concept of induced membrane for reconstruction of long bone defects. Orthop Clin North Am 2010;41:27-37.

7. Germain MA, Mascard E, Dubousset J, Nguefack M. Free vascularized fibula and reconstruction of long bones in the child--our evolution. MICROSURG 2007;27:415-9.

8. Friedrich JB, Moran SL, Bishop AT, Wood CM, Shin AY. Free vascularized fibular graft salvage of complications of long-bone allograft after tumor reconstruction. J BONE JOINT SURG AM 2008;90:93100.

9. Tetsworth K, Paley D, Sen C, Jaffe M, Maar DC, Glatt V, et al. Bone transport versus acute shortening for the management of infected tibial non-unions with bone defects. INJURY 2017;48:2276-84.

10. Pipitone PS, Rehman S. Management of traumatic bone loss in the lower extremity. Orthop Clin North Am 2014;45:469-82.

11. Szabo RM, Anderson KA, Chen JL. Functional outcome of en bloc excision and osteoarticular allograft replacement with the Sauve-Kapandji procedure for Campanacci grade 3 giant-cell tumor of the distal radius. J Hand Surg Am 2006;31:1340-8.

12. Pirela-Cruz MA, DeCoster TA. Vascularized bone grafts. ORTHOPEDICS 1994;17:407-12.

13. Morris R, Hossain M, Evans A, Pallister I. Induced membrane technique for treating tibial defects gives mixed results. BONE JOINT J 2017;99-B:680-5.

14. Wu Y, Yin Q, Rui Y, Sun Z, Gu S. Ilizarov technique: Bone transport versus bone shortening-lengthening for tibial bone and soft-tissue defects. J ORTHOP SCI 2018;23:341-5.

15. Catagni MA, Azzam W, Guerreschi F, Lovisetti L, Poli P, Khan MS, et al. Trifocal versus bifocal bone transport in treatment of long segmental tibial bone defects. BONE JOINT J 2019;101-B:162-9.

16. Aktuglu K, Gunay H, Alakbarov J. Monofocal bone transport technique for bone defects greater than 5 cm in tibia: our experience in a case series of 24 patients. INJURY 2016;47 Suppl 6:S40-6. 
17. Baumgart R, Schuster $B$, Baumgart $T$. [Callus distraction and bone transport in the treatment of bone defects]. ORTHOPADE 2017;46:673-80.

18. Vesely R, Prochazka V. [Callus Distraction in the Treatment of Post-Traumatic Defects of the Femur and Tibia]. Acta Chir Orthop Traumatol Cech 2016;83:388-92.

19. Aktuglu K, Erol K, Vahabi A. Ilizarov bone transport and treatment of critical-sized tibial bone defects: a narrative review. J Orthop Traumatol 2019;20:22.

20. Jiang Q, Huang K, Liu Y, Chi G. Using the llizarov technique to treat limb shortening after replantation of a severed lower limb: a case report. Ann Transl Med 2020;8:1025.

21. Zhang Y, Wang Y, Di J, Peng A. Double-level bone transport for large post-traumatic tibial bone defects: a single centre experience of sixteen cases. INT ORTHOP 2018;42:1157-64.

22. Yushan M, Ren P, Abula A, Alike Y, Abulaiti A, Ma C, et al. Bifocal or Trifocal (Double-Level) Bone Transport Using Unilateral Rail System in the Treatment of Large Tibial Defects Caused by Infection: A Retrospective Study. ORTHOP SURG 2020;12:184-93.

23. Borzunov DY. Long bone reconstruction using multilevel lengthening of bone defect fragments. INT ORTHOP 2012;36:1695-700.

24. Paley D, Catagni MA, Argnani F, Villa A, Benedetti GB, Cattaneo R. llizarov treatment of tibial nonunions with bone loss. Clin Orthop Relat Res 1989:146-65.

25. Paley D. Problems, obstacles, and complications of limb lengthening by the llizarov technique. Clin Orthop Relat Res 1990:81-104.

26. Kocaoglu M, Eralp L, Rashid HU, Sen C, Bilsel K. Reconstruction of segmental bone defects due to chronic osteomyelitis with use of an external fixator and an intramedullary nail. J BONE JOINT SURG AM 2006;88:2137-45.

27. Ilizarov GA. The tension-stress effect on the genesis and growth of tissues. Part I. The influence of stability of fixation and soft-tissue preservation. Clin Orthop Relat Res 1989:249-81.

28. Mahaluxmivala J, Nadarajah R, Allen PW, Hill RA. llizarov external fixator: acute shortening and lengthening versus bone transport in the management of tibial non-unions. INJURY 2005;36:662-8.

29. Gupta S, Malhotra A, Mittal N, Garg SK, Jindal R, Kansay R. The management of infected nonunion of tibia with a segmental defect using simultaneous fixation with a monorail fixator and a locked plate. BONE JOINT J 2018;100-B:1094-9.

30. Gulabi D, Erdem M, Cecen GS, Avci CC, Saglam N, Saglam F. llizarov fixator combined with an intramedullary nail for tibial nonunions with bone loss: is it effective? Clin Orthop Relat Res 
31. Kocaoglu M, Eralp L, Rashid HU, Sen C, Bilsel K. Reconstruction of segmental bone defects due to chronic osteomyelitis with use of an external fixator and an intramedullary nail. J BONE JOINT SURG AM 2006;88:2137-45.

32. Sala F, Thabet AM, Castelli F, Miller AN, Capitani D, Lovisetti G, et al. Bone transport for postinfectious segmental tibial bone defects with a combined ilizarov/taylor spatial frame technique. J ORTHOP TRAUMA 2011;25:162-8.

33. Borzunov DY, Chevardin AV. Ilizarov non-free bone plasty for extensive tibial defects. INT ORTHOP 2013;37:709-14.

\section{Tables}

Table 1 Demographics of the two groups

\begin{tabular}{|c|c|c|c|c|}
\hline Parameter & BFT group & TFT group & Statistical value & P-value \\
\hline Patients & 32 & 21 & - & - \\
\hline \multicolumn{5}{|l|}{ Gender } \\
\hline Male & 23 & 16 & \multirow{2}{*}{0.121} & \multirow{2}{*}{0.727} \\
\hline Female & 9 & 5 & & \\
\hline Age (year) & $38.2 \pm 12.3$ & $39.9 \pm 12.9$ & -0.479 & 0.634 \\
\hline \multicolumn{5}{|l|}{ Etiology of bone defect } \\
\hline Infected nonunion & 10 & 7 & \multirow[t]{2}{*}{0.025} & \multirow{2}{*}{0.874} \\
\hline Chronic osteomyelitis & 22 & 14 & & \\
\hline \multicolumn{5}{|l|}{ Injured tibia } \\
\hline Left & 13 & 9 & \multirow[t]{2}{*}{0.026} & \multirow{2}{*}{0.872} \\
\hline Right & 19 & 12 & & \\
\hline \multicolumn{5}{|l|}{ Location of bone defect } \\
\hline Proximal & 5 & 3 & \multirow{2}{*}{0.164} & \multirow[t]{2}{*}{0.921} \\
\hline Middle & 18 & 11 & & \\
\hline $\begin{array}{l}\text { Distal } \\
\text { Mean previous operation time }\end{array}$ & $\begin{array}{c}9 \\
25+09\end{array}$ & $\begin{array}{c}7 \\
2.6+1.1\end{array}$ & -0.307 & 0.760 \\
\hline $\begin{array}{l}\text { Mean previous operation time } \\
\text { Mean defect size }(\mathrm{cm})\end{array}$ & $\begin{array}{l}2.5 \pm 0.9 \\
7.8 \pm 1.8\end{array}$ & $\begin{array}{l}2.6 \pm 1.1 \\
9.4 \pm 1.5\end{array}$ & -3.340 & 0.002 \\
\hline BFT: bifocal bone transport. TH & - trifolal & 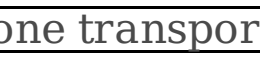 & & \\
\hline
\end{tabular}

Table 2 Comparison of the results in the two groups

\begin{tabular}{lcccc}
\hline Parameter & BFT group & TFT group & Statistical value & P-value \\
\hline Mean OD (mins) & $122.5 \pm 11.2$ & $161.9 \pm 8.9$ & -13.544 & $\mathrm{P}<0.001$ \\
Mean DT (days) & $96.8 \pm 22.6$ & $65.9 \pm 10.8$ & 5.833 & $\mathrm{P}<0.001$ \\
Mean EFT (days) & $474.5 \pm 103.2$ & $328.0 \pm 57.2$ & 5.919 & $\mathrm{P}<0.001$ \\
Mean EFI (days/cm) & $60.8 \pm 1.9$ & $34.8 \pm 2.1$ & 46.492 & $\mathrm{P}<0.001$ \\
\hline
\end{tabular}

BFT: bifocal bone transport. TFT: trifocal bone transport. OD: operation duration DT: docking time. EFT: external fixation time. EFI: external fixation index 
Table 3 Results of ASAMI scores

\begin{tabular}{ccccccc}
\hline Parameter & Excellent & Good & Fair & Poor & Failure & P-value \\
\hline Bone results & 13 & 15 & 3 & 1 & - & 0.903 \\
BFT group & 11 & 8 & 2 & 0 & - & \\
TFT group & 10 & 16 & 6 & 0 & 0 & 0.844 \\
Functional results & 8 & 10 & 3 & 0 & 0 & \\
BFT group & & & &
\end{tabular}

BFT: bifocal bone transport. TFT: trifocal bone transport.

ASAMI Criteria:

Bone results

Excellent: Union, no infection, deformity $<7^{\circ}$, limb length discrepancy (LLD) $<2.5 \mathrm{~cm}$

Good: Union plus any two of the following: absence of infection, deformity $<7^{\circ}$, LLD $<2.5 \mathrm{~cm}$.

Fair: Union plus any one of the following: absence of infection, deformity $<7^{\circ}, \mathrm{LLD}<2.5 \mathrm{~cm}$.

Poor: Nonunion/refracture/union plus infection plus deformity $>7^{\circ}$ plus LLD $>2.5 \mathrm{~cm}$

Functional results

Excellent: Active, no limp, minimum stiffness (loss of $<15^{\circ} \mathrm{knee}$ extension $/<15^{\circ}$ ankle dorsiflexion) no reflex sympathetic dystrophy (RSD), insignificant pain.

Good: Active, with one or two of the following: limb, stiffness, RSD, significant pain

Fair: Active, with three or all of the following: limb, stiffness, RSD, significant pain

Poor: Inactive (unemployment or inability to return to daily activities because of injury)

Failure: Amputation

Table 4 Difficulties during the treatment in the two groups

\begin{tabular}{llll}
\hline Difficulty & BFT group $(\mathrm{n}=32)$ & TFT group $(\mathrm{n}=21)$ & P-value \\
\hline Problem & 43 & 17 & \\
Obstacle & 20 & 8 & \\
Complication & 31 & 11 & \\
Total difficulties & 94 & 36 & 0.001 \\
Mean difficulties/patient & 2.9 & 1.7 & \\
\hline BFT: bifocal bone transport. TFT: trifocal bone transport & \\
\hline
\end{tabular}

Table 5 Summary of complications in the two groups

\begin{tabular}{|c|c|c|c|c|c|}
\hline \multirow[b]{2}{*}{ Complications } & \multicolumn{2}{|c|}{ BFT group $(n=32)$} & \multicolumn{2}{|c|}{ TFT group $(n=21)$} & \multirow[b]{2}{*}{ P-value } \\
\hline & Number & Percentage & Number & Percentage & \\
\hline Pin tract infection & 5 & $15.6 \%$ & 2 & $9.5 \%$ & \\
\hline Axial deviation & 7 & $21.9 \%$ & 3 & $14.3 \%$ & \\
\hline Soft tissue incarceration & 3 & $9.4 \%$ & 1 & $4.8 \%$ & \\
\hline Joint stiffness & 11 & $34.4 \%$ & 5 & $23.8 \%$ & \\
\hline Delayed union & 4 & $12.5 \%$ & 0 & $0.0 \%$ & \\
\hline Nonunion & 1 & $3.1 \%$ & 0 & $0.0 \%$ & \\
\hline Refracture & 0 & $0.0 \%$ & 0 & $0.0 \%$ & \\
\hline Total complications & \multicolumn{2}{|r|}{31} & \multicolumn{2}{|r|}{11} & \\
\hline Mean complications/patient & \multicolumn{2}{|r|}{1.0} & \multicolumn{2}{|r|}{0.5} & 0.032 \\
\hline
\end{tabular}

\section{Figures}



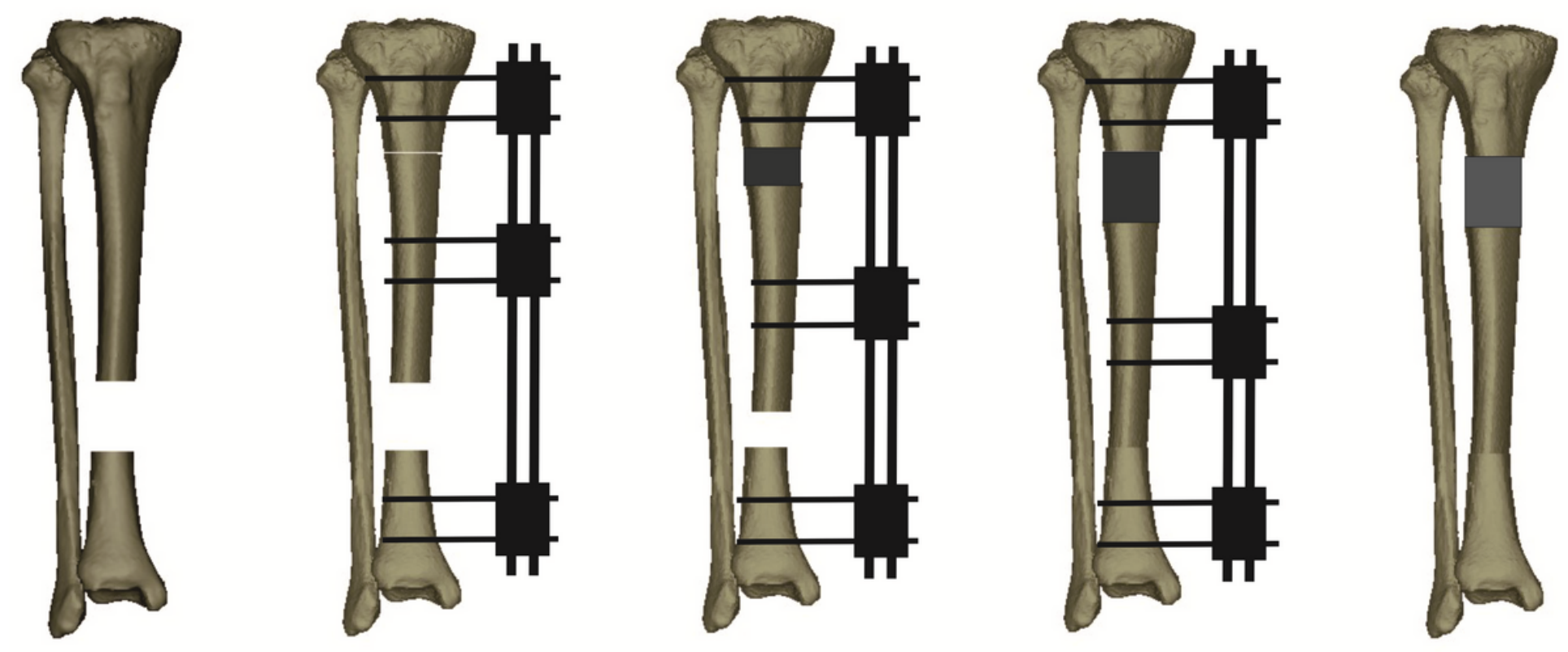

\section{A}
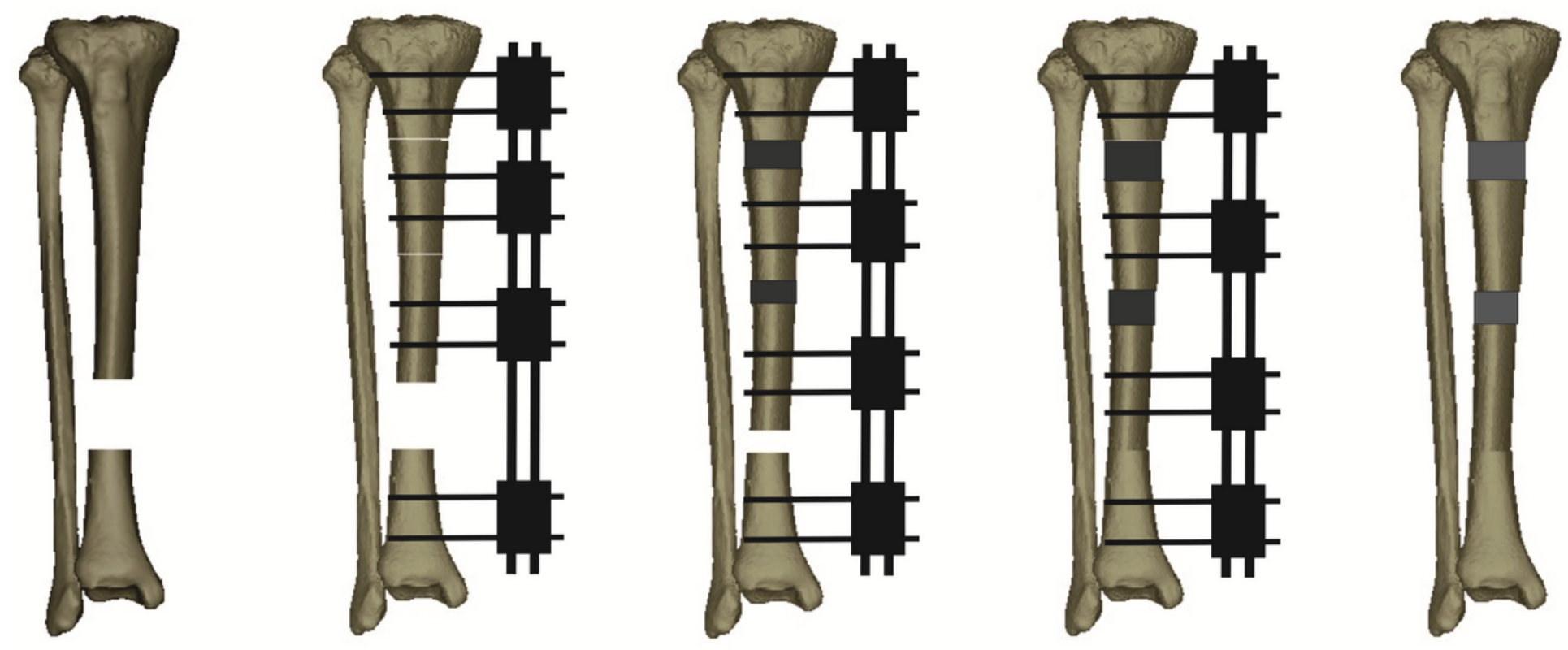

Figure 1

Schematic diagram of the two bone transport techniques for the management of the lower third bony segmental defects in the tibia (from left to right). a Bifocal bone transport technique. $b$ Trifocal bone transport technique. 

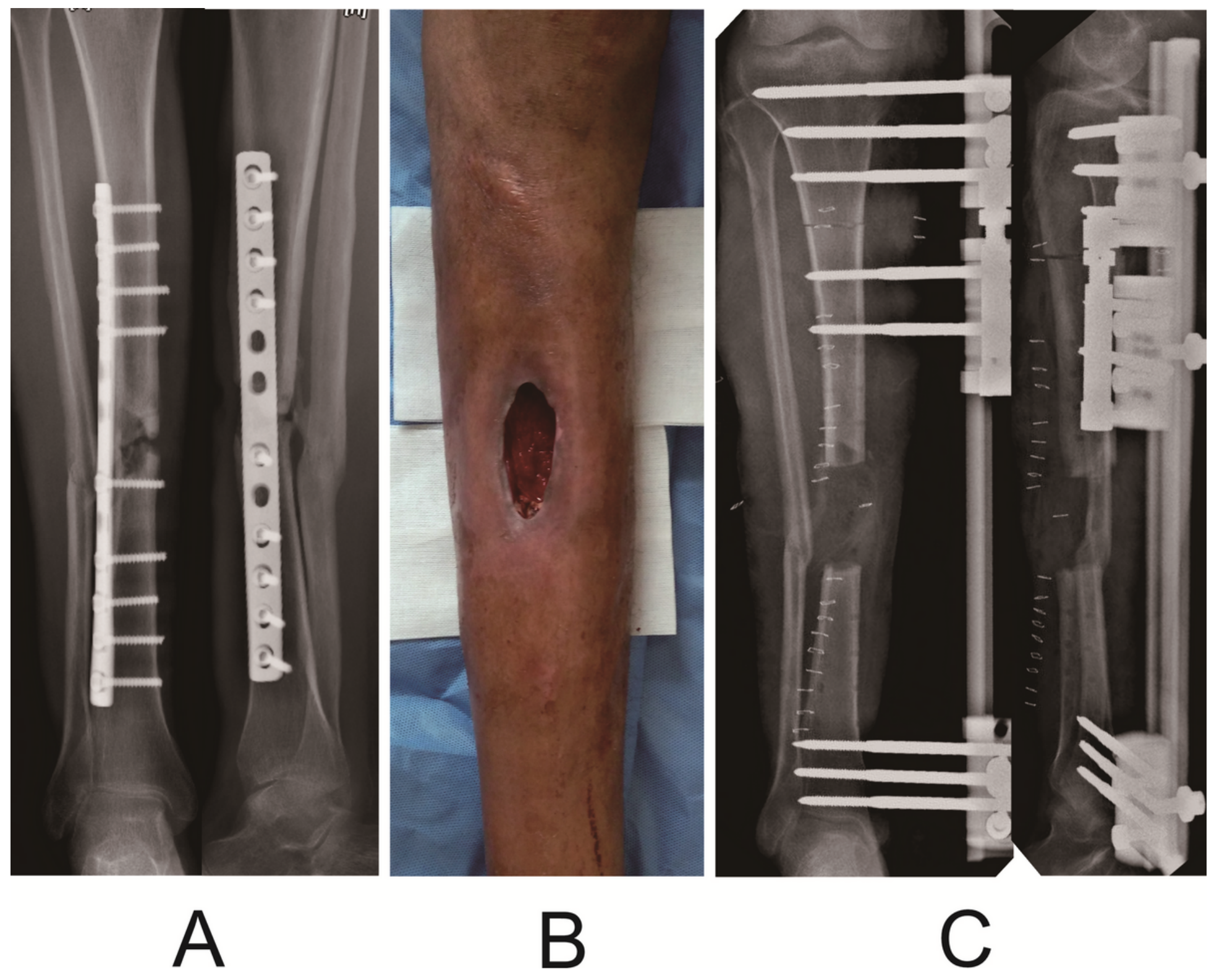

Figure 2

A 42-year-old man who suffered chronic osteomyelitis in his right tibia after internal fixation treatment followed by a road traffic accident and successfully treated by bifocal bone transport technique from proximal to distal. a Preoperative AP and lateral radiographs. b Preoperative general appearance, showing soft tissue defects with drainage and sinus. c AP and lateral radiographs immediately after radical debridement and installation of monolateral external fixator, there were $6 \mathrm{~cm}$ bone defects. $A$ bifocal bone transport technique from proximal to distal was performed to reconstruct the injured limb. 


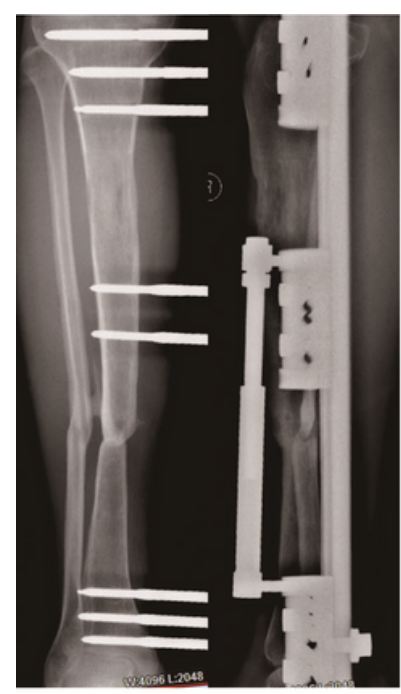

A

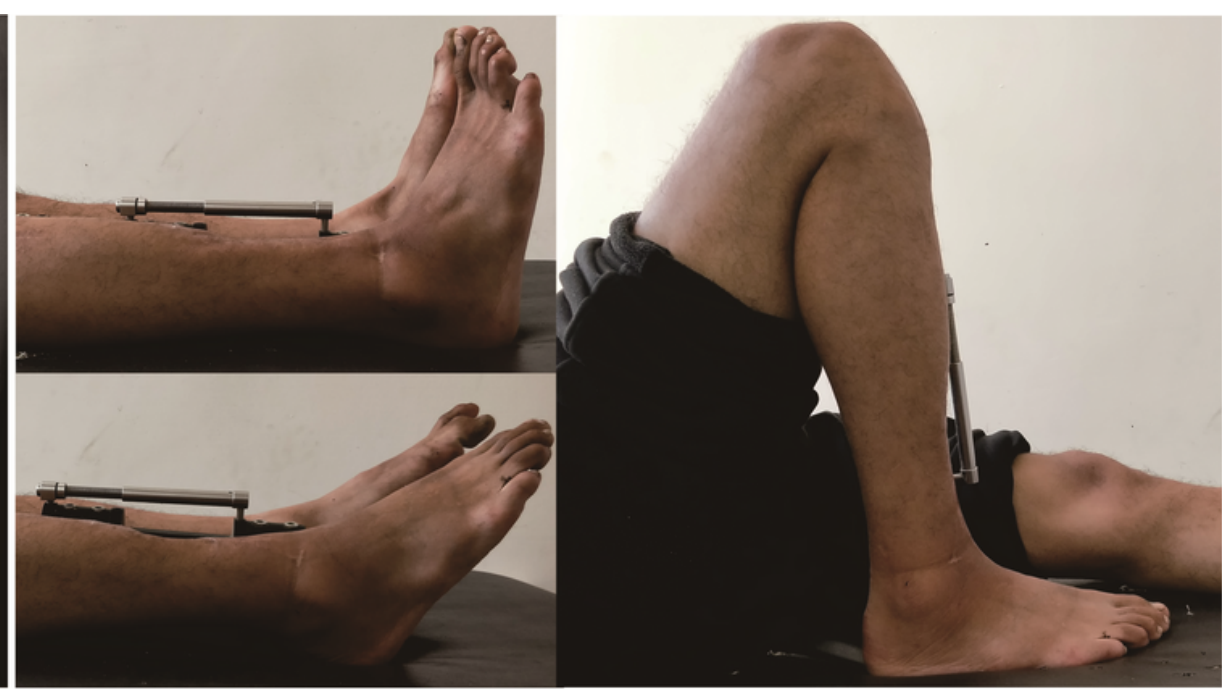

$\mathrm{B}$

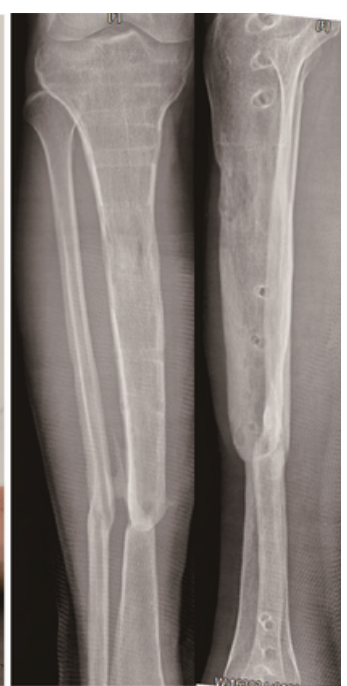

C

Figure 3

Images of the same patient shown in Figure 2. a Radiographs manifest the complete consolidation and docking site union. $b$ General appearance before frame removal, showing the satisfactory range of motion of knee and ankle joint results. c Radiographs six months later after removing the external fixator.

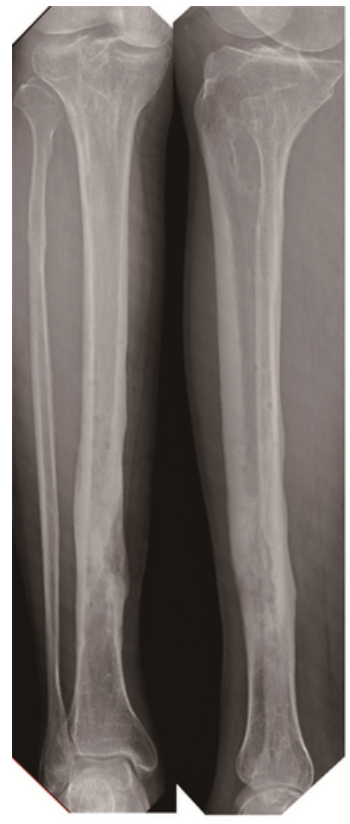

A

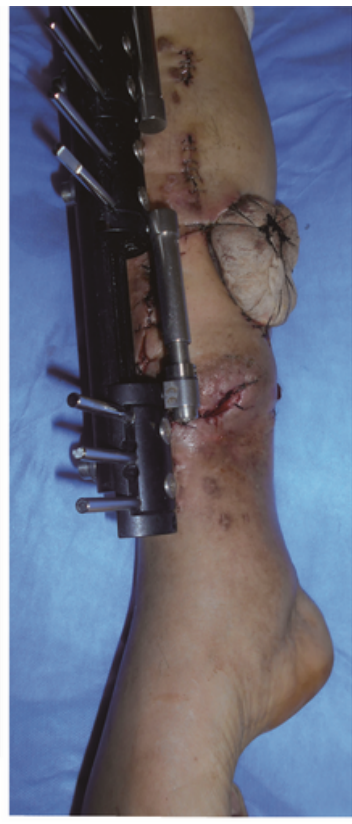

B

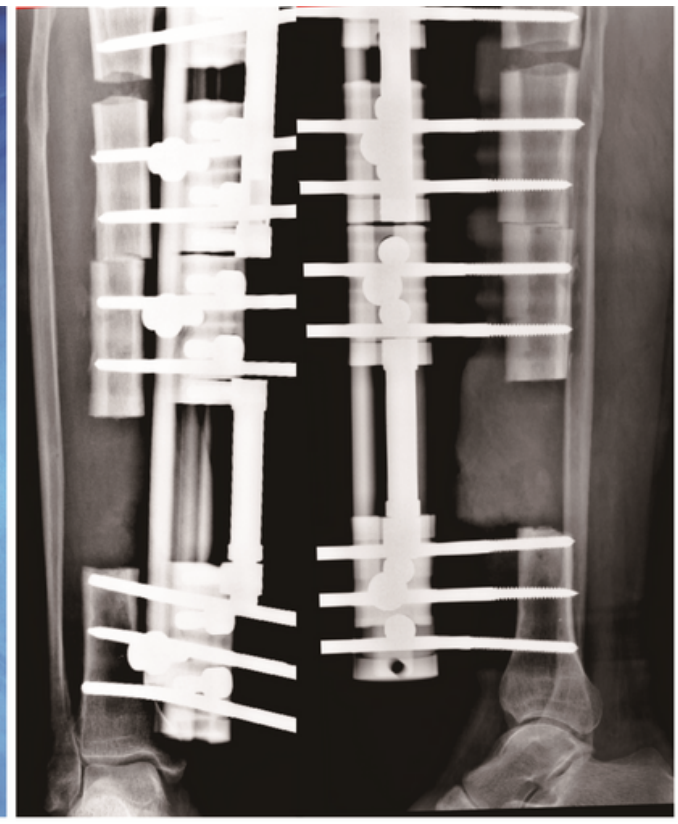

C

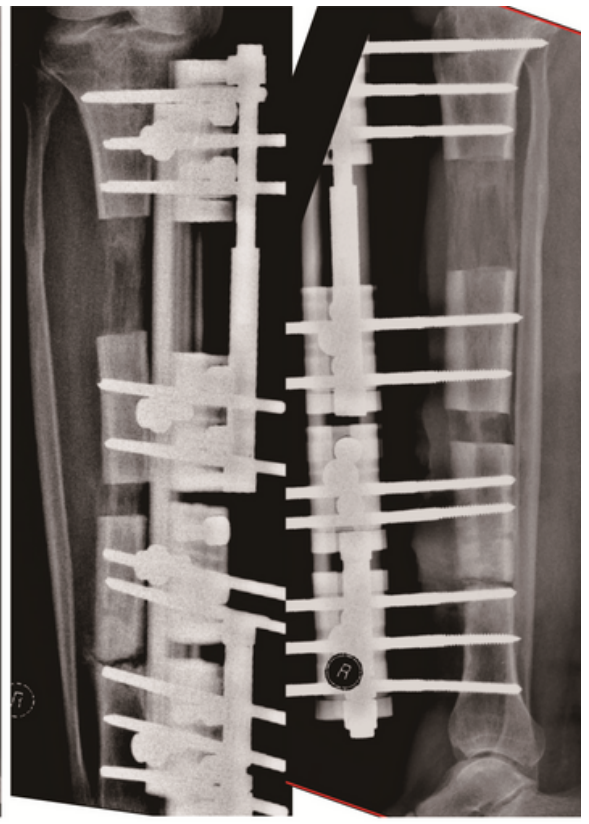

$\mathrm{D}$

Figure 4

A 53-year-old man suffered chronic osteomyelitis in his right tibia after internal fixation treatment due to a crushing injury caused by a heavy object and successfully managed by trifocal bone transport technique (tandem transport, from proximal to distal). a Preoperative radiographs indicated that the infectious lesion was located at the distal $1 / 3$ of the tibial shaft. b Removal of devitalized bone and soft tissue by radical debridement, the soft tissue defect was treated by a local tissue flap. c There were $9 \mathrm{~cm}$ bone 
defects, and a trifocal tandem bone transport from proximal to distal was conducted for the limb reconstruction.

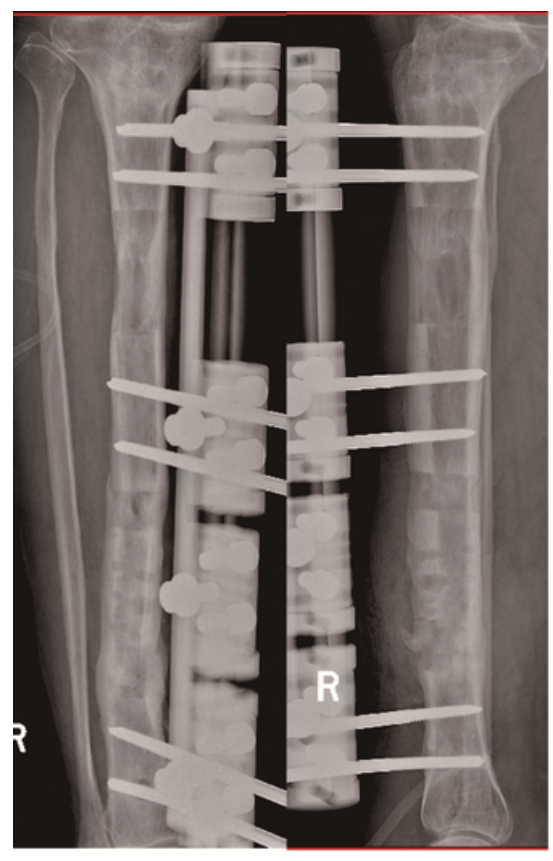

A

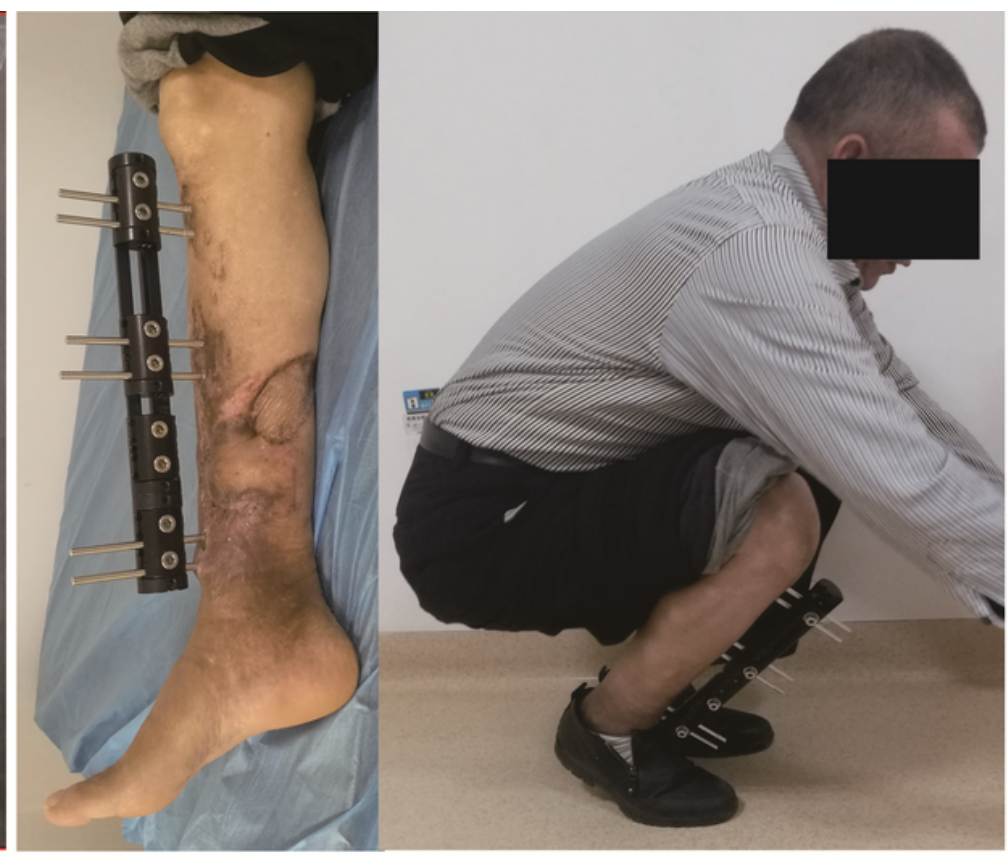

B

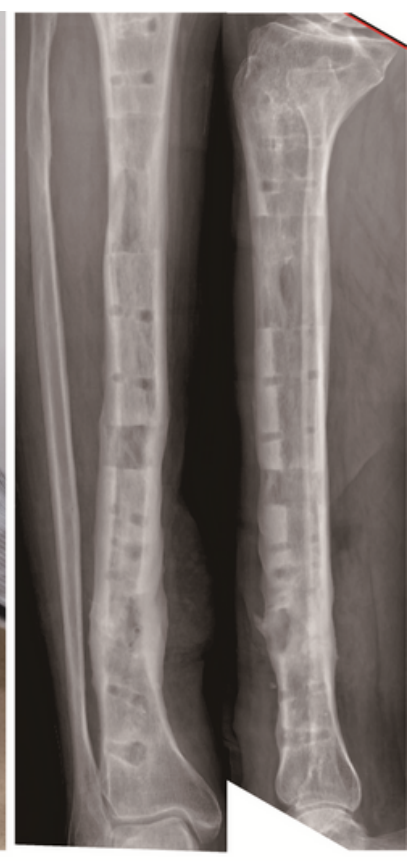

C

\section{Figure 5}

Images of the same patient shown in Figure 4. a Complete consolidation and docking site union after docking in three months. $b$ Satisfactory functional recovery before the monolateral external fixator removal. c Radiographs nine months later after removing the frame. 